\title{
Modeling of Electron Transpiration Cooling for Hypersonic Vehicles
}

\author{
Kyle M. Hanquist* \\ University of Michigan, Ann Arbor, MI, 48109, USA \\ Kentaro $\mathrm{Hara}^{\dagger}$ \\ Princeton Plasma Physics Laboratory, Princeton, NJ, 08543, USA \\ Iain D. Boyd ${ }^{\ddagger}$ \\ University of Michigan, Ann Arbor, MI, 48109, USA
}

\begin{abstract}
Electron transpiration cooling (ETC) is a recently proposed approach to manage the high heating loads experienced at the sharp leading edges of hypersonic vehicles. Computational fluid dynamics can be used to investigate the feasibility of ETC in a hypersonic environment. A modeling approach is presented for ETC, which includes devloping the boundary conditions for electron emission from the surface, accounting for the electric field and space-charge limit effects within the near-wall plasma sheath. Two different analytical models for space-charge limited emission are discussed. The first model assumes that the electrons are emitted cold from the surface while in the second approach the emitted electrons have a finite temperature. The theory shows that emitted electrons with a finite temperature, referred to as warm emission in the present paper, can reach higher levels of emission. This is important because the benefit of ETC, mainly reduction in the surface temperature, is directly correlated to the level of electron emission from the surface. The space-charge limit models are assessed using 1D direct-kinetic plasma sheath simulations. The simulations agree well with the space-charge limit theory proposed by Takamura et al. for emitted electrons with a finite temperature. Both models are implemented into a CFD code, LeMANS, and run for a test case typical of a leading edge radius in a hypersonic flight environment. The CFD results show finite temperature theory results in a larger reduction in wall temperature because more electron emission is allowed for than the cold emission theory. However, even with the electrons being emitted with a finite temperature, the emission still reaches space-charge limits for the test case considered, which can limit the benefits of ETC.
\end{abstract}

\section{Nomenclature}

$A_{R} \quad$ Richardson constant, $1.20 \times 10^{6} \mathrm{~A} / \mathrm{m}^{2} / \mathrm{K}^{2}$

$\bar{C} \quad$ Thermal speed

E Electric field

j Electric current density

$J \quad$ Current density

$k_{B} \quad$ Boltzmann constant, $1.38 \times 10^{-23} \mathrm{~J} / \mathrm{K}$

$m_{s} \quad$ Mass of species $s$

$M_{s} \quad$ Molar mass of species $s$

$n \quad$ Number density

$N_{A v}$ Avogadro constant, $6.02 \times 10^{23} \mathrm{~mol}^{-1}$

*PhD Candidate, Department of Aerospace Engineering, Ann Arbor, MI, 48109, USA, AIAA Student Member.

$\dagger$ Visiting Research Physicist, Princeton Plasma Physics Laboratory, Princeton, NJ, 08543, USA.

${ }^{\ddagger}$ James E. Knott Professor, Department of Aerospace Engineering, Ann Arbor, MI, 48109, USA, AIAA Fellow. 


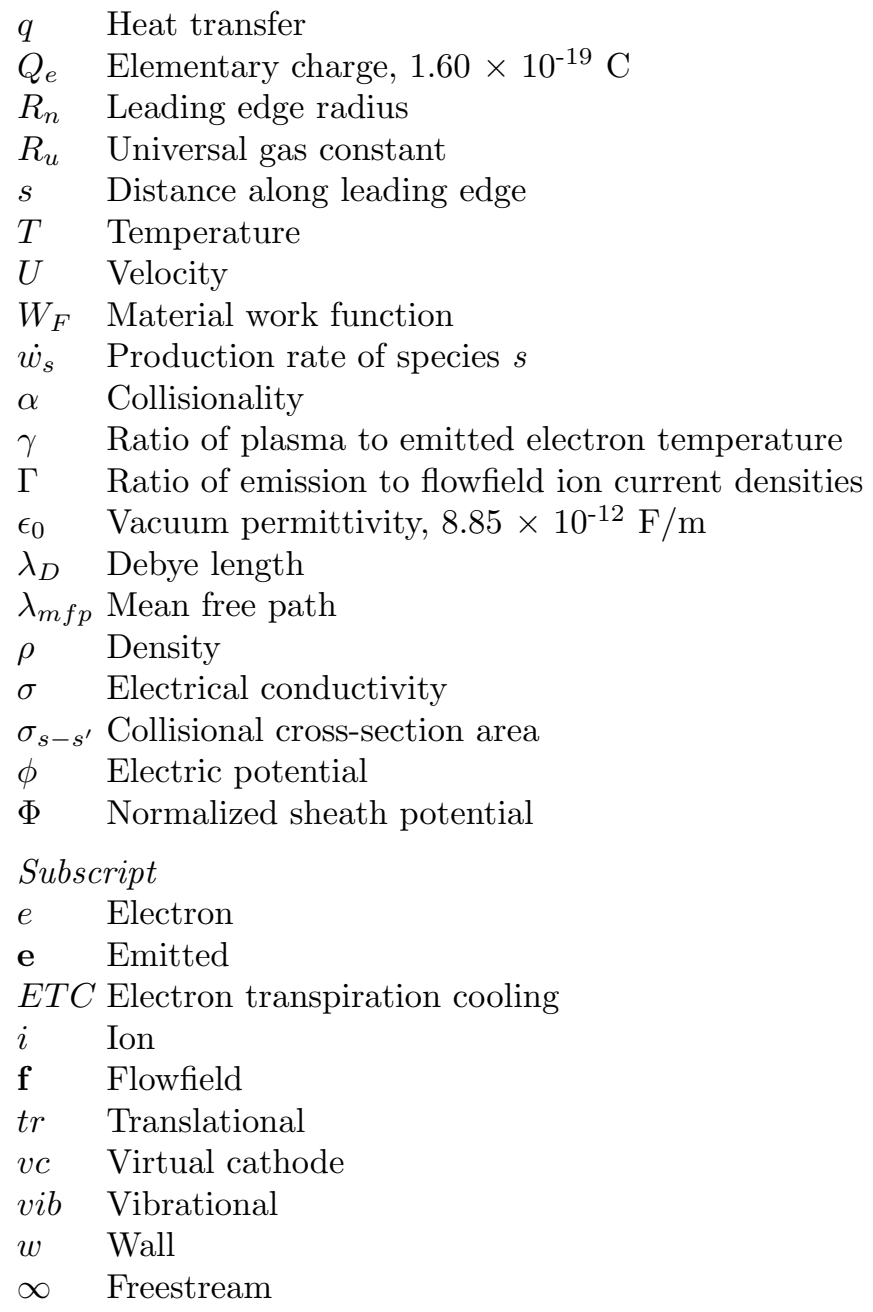

\section{Introduction}

7 HE development of aeronautics has been largely driven by the passion to fly faster, and recent advance1 ments in technologies have enabled the possibility of hypersonic cruise vehicles. These vehicles have a sharp leading edge radius to increase the lift-to-drag ratio. However, the aerodynamic performance gains obtained by having a sharp leading edge come at the cost of very high, localized heat rates. A theoretical analysis first performed by Fay and Riddell ${ }^{1}$ reveals that the stagnation point heat transfer in hypersonic flow is inversely proportional to the radius of the vehicle leading edge,

$$
q_{w} \propto \sqrt{\frac{\rho_{\infty}}{R_{n}}} U_{\infty}^{3}
$$

Equation 1 shows that as these vehicles continue to fly faster and farther, managing these increasing heat loads will be important. There are three types of thermal management for these heat loads: passive, semipassive, and active. A passive thermal management system utilizes a material that could cool the surface through radiation while minimizing the heat flow to the structure and withstand a high temperature. A type of material that meets this criteria is ulta-high temperature composite (UHTC) materials, which were used on the NASA X-43 experimental aircraft that flew at Mach 9.2. ${ }^{2}$ Although UHTC materials have good thermal properties, they have some physical limitations such as weak fracture toughness and heavy weight that suggest they are not the ideal approach. ${ }^{3}$ Equation 1 shows stagnation point heat transfer has a cubic relation with freestream velocity. So as these vehicles fly faster, passive thermal management will not be sufficient to manage the immense heat loads. Another approach is to use a semi-passive or active thermal management system. These approaches are explained in detail in Ref. 4. Semi-passive approaches involve managing the heat through a heat pipe or ablation. But, in order to reduce the drag, these vehicles 
will have a sharp leading edge radius, which limits the spacing for heat pipes within the leading edge. Ablating materials have significant heat management benefits through essentially a controlled melt of the heat shield, which help moves the heat into the flow as opposed to into the surface. This shape change of the surface, while permitted for the blunt bodies of re-entry flight, is unacceptable for the sharp leading edges of hypersonic vehicles. As hypersonic vehicles continue to fly faster and farther, resulting in higher heat fluxes for longer durations, active cooling will be required. These approaches include convective cooling, film cooling, and transpiration cooling. Convective cooling involves the heat being transferred to a coolant flow within the structure but this will be limited by the size of the leading edge radius and is mostly used for propulsion system heat management. Film cooling and transpiration cooling involves injecting a coolant into the flow either through an injection point upstream of the high heat loads (film cooling used in propulsion systems) or utilizing a porous structure where the coolant is injected through the pores continuously over large areas (transpiration cooling). A drawback to transpiration cooling, in this definition, at the leading edges of hypersonic vehicles would be the coolant flow through the pores could be difficult at the stagnation point due to the high stagnation pressure.

An alternative approach that has been proposed recently involves using thermionic materials at the leading edges of hypersonic vehicles. ${ }^{5}$ When exposed to high convective heating rates, these materials emit a current of electrons that leads to a transpiration cooling effect of the surface of the vehicle called electron transpiration cooling (ETC). This transpiration cooling effect would not require a porous material as the electrons would be emitted from the material surface. The electrons are emitted through a phenomenon known as thermionic emission that occurs when the thermal energy given to the electrons is greater than the binding potential of the surface material. The binding potential of the material is defined as the work function. A material with a lower work function would require less thermal energy for an electron to be emitted. Candidate materials for ETC have a low work function $(2-3 \mathrm{eV})$ and are currently being developed. A conceptual study was completed and showed that ETC is more beneficial for higher freestream velocities, sharper leading edge radii, and lower material work functions. ${ }^{6}$ The modeling approach for ETC was recently compared against a set of experiments and agreed well given the uncertainties in the experiments. ${ }^{7}$

Given the promising trends of ETC in previous work, this paper focuses on improving the modeling capabilities of ETC. It has been shown that the benefits of ETC are directly tied to the level of emission from the surface, ${ }^{8}$ so a focus will be on accurately modeling how many electrons leave the surface. First, a description of the numerical methods used and test case conditions are outlined. Then a comparison between space-charge limited theory and direct-kinetic solutions are presented. The theory is then implemented into the CFD code, LeMANS, which is used to investigate the effects the space-charge limits have on a test case typical of hypersonic flight. Finally, the paper ends with some conclusions drawn from this study and outlines future work recommendations.

\section{Numerical Approach}

\section{A. Computational Fluid Dynamics}

The numerical simulations in this work are performed using the CFD code LeMANS, which was developed at the University of Michigan. ${ }^{9}$ LeMANS is a parallel, three-dimensional code that solves the Navier-Stokes equations on unstructured computational grids. LeMANS includes thermo-chemical non-equilibrium effects and the flow is modeled assuming that the continuum approximation is valid. It is also assumed that the translational and rotational energy modes can be described by a single temperature, $T_{t r}$, and that the vibrational, electronic, and electron translational energy modes are described by a different temperature, $T_{v i b}$. The mixture transport properties are calculated using Wilke's semi-empirical mixing rule ${ }^{10}$, species thermal conductivities determined using Eucken's relation, ${ }^{11}$ and species viscosities determined using Blottner's curve fits. ${ }^{12}$ A standard finite-rate chemistry model is used for eleven species reactive air, and Park's two-temperature model ${ }^{13}$ is used to account for the effects of thermal non-equilibrium on the forward and backward reactions rates. In LeMANS, the set of governing equations is solved using the finite-volume method applied to unstructured grids with second-order spatial accuracy. A modified Steger-Warming Flux Vector Splitting scheme ${ }^{14}$ is used to discretize the inviscid fluxes across cell faces. The viscous terms are computed using cell-centered and nodal values. The viscous stresses are modeled assuming the flow is a Newtonian fluid and Stokes' hypothesis is applicable, and the heat fluxes are modeled according to Fourier's law for all temperatures. 


\section{Electron Emission}

The electron current density is a function of the material's surface temperature and work function as determined by Richardson, ${ }^{15}$

$$
J_{e, \mathbf{e}}=A_{R} T_{w}^{2} \exp \left(\frac{-W_{F} Q_{e}}{k_{B} T_{w}}\right)
$$

which shows that materials with lower work functions and higher surface temperatures will result in more electron emission. It is to be noted that this current is only realized in ideal conditions: emitted electrons see no retarding electric field at the surface, are not reflected back to the surface through collisions, or see a virtual cathode created by space-charge limits. The hypersonic flow environment near the leading edges are not typical of the aforementioned ideal conditions and the effect these real hypersonic emitter conditions have on ETC are discussed later. A boundary condition is implemented into LeMANS to model the production rate of electrons,

$$
\dot{w}_{e}=\frac{J_{e, \mathbf{e}}}{Q_{e} N_{A v}}-\sum_{s=i o n s} \dot{w}_{s}
$$

where $\dot{w}_{s}$ is the surface recombination rate of ions. The recombination rate of ions is calculated assuming that the surface is fully catalytic to ions: ${ }^{16}$

$$
\dot{w}_{i}=\frac{\rho_{i}}{M_{i}} \sqrt{\frac{R_{u} T_{w}}{2 \pi M_{i}}}
$$

The emitted electrons will carry away energy from the vehicle surface determined by the electrons overcoming the potential barrier and the kinetic energy associated with the emitted electrons resulting in a heat flux of, ${ }^{15}$

$$
q_{E T C}=J_{e, \mathbf{e}}\left(\Phi+\frac{2 k_{B} T_{w}}{Q_{e}}\right)
$$

The surface temperature is determined by balancing the heat going into the surface (convective heat transfer) to the heat leaving the surface (radiation and ETC). It is to be noted that in-depth surface conduction is not considered in the current work as well as the effect of negatively biasing the surface on the heat transfer balance (ions will accelerate into the surface and electrons will accelerate into the plasma) and will be investigated in future work.

\section{Electric Field}

Given that a large current of electrons will be emitted from the leading edge, LeMANS is modified in order to account for the electric field, to some extent. The electric field can be replaced by an electric potential, $\phi$, as,

$$
\vec{E}=-\nabla \phi
$$

where the electric potential is calculated using Ohm's law and solving the steady state current continuity equation,

$$
\begin{gathered}
\vec{j}=\sigma \vec{E} \\
\nabla \cdot \vec{j}=\nabla \cdot(\sigma \nabla \phi)=0
\end{gathered}
$$

where $\sigma$ is the electrical conductivity of the plasma. The electrical conductivity in this work is approximated using a semi-empirical model developed by Razier,${ }^{17}$ which is valid for air, nitrogen, and argon,

$$
\sigma=8300 \times \exp \left(\frac{-36,000}{T}\right)
$$

A zero-gradient condition is used at the inlet, outlet, and symmetry computational boundaries for the electric potential. At the wall, the electric potential is driven by the plasma sheath physics discussed in Section 3. The electric field at the wall can decrease the energy barrier that the emitted electrons must overcome at the surface, which effectively reduces the work function, known as the Schottky effect: ${ }^{18}$

$$
W_{F, c}=W_{F}-\sqrt{\frac{Q_{e}^{3} E_{w}}{4 \pi \epsilon_{0}}}
$$


where $E_{w}$ is the electric field at the wall and $\epsilon_{0}$ is the vacuum permittivity. Previous work showed that the magnitude of the electric field for cases similar to this study has a relatively small value, which causes negligible effects on the predicted surface properties of hypersonic vehicles with thermo-electric TPS materials, ${ }^{6}$ but will play an important role in future work where electrons must reattach downstream on the aftbody to prevent the vehicle from accumulating a net charge.

\section{Plasma Sheath}

A plasma sheath forms near the the wall, which is a non-neutral region between the quasineutral flowfield and the wall. The sheath occurs because the electrons are much more mobile than the ions due to their mass difference $\left(m_{e} \ll m_{i}\right)$. This higher mobility of electrons leads to more electrons leaving this region than ions, leaving the region positively charged, which generates a negative potential difference between the flowfield and the wall. The length scale of this non-neutral region is on the order of the Debye length:

$$
\lambda_{D}=\sqrt{\frac{\epsilon_{0} k_{B} T_{e}}{Q_{e}^{2} n_{e}}}
$$

An assumption made in LeMANS is that at the wall, all the temperatures equal the wall temperature $\left(T_{e}=T_{i}=T_{t r}=T_{w}\right)$. This is an underestimation of the electron temperature but it has been shown that the electron temperature is on the order of magnitude of the translational and vibrational temperature in a typical hypersonic flight environment. ${ }^{19}$ Another important sheath parameter is the sheath collisionality, which is proportional to the number of collisions that occur within the sheath,

$$
\alpha \equiv \frac{\lambda_{D}}{\lambda_{m f p}}
$$

where $\lambda_{m f p}$ is the mean free path. The mean free paths of interest are the ion-neutral and electron-neutral mean free paths:

$$
\lambda_{m f p, i-n}=\frac{1}{\left(n_{i}+n_{\text {neut }}\right) \sigma_{i}}, \quad \lambda_{m f p, e-n}=\frac{1}{\left(n_{e}+n_{\text {neut }}\right) \sigma_{e}}
$$

where $\sigma_{i}$ and $\sigma_{e}$ are the collisional cross sections of the ions and electrons, respectively. For the cases of interest, the Debye length $(\leq 10 \mu \mathrm{m})$ is smaller than the ion-neutral and electron-neutral mean free paths $(\geq 100 \mu \mathrm{m})$, which leads to the collisionality of the sheath being smaller than one. If $\alpha \ll 1$, the sheath can be treated as collisionless. ${ }^{20}$ For this work, the sheath is assumed to be collisionless, although there could be some collisions within the sheath since the collisionality is not much smaller than one. A previous study showed that the sheath width is four to ten times the Debye length so $\alpha$ can be on order of one. ${ }^{21}$ The effect of sheath collisionality will be investigated in future work.

Previous work investigated treating the emitter surface as either an electronically floated surface or a negatively biased surface and it was shown in order for ETC to be beneficial, the surface should be negatively biased. ${ }^{8}$ A surface such as this would occur if the emitted electrons reattach downstream on the vehicle and travel back to the emitter surface completing the circuit. This work assumes that the surface can be biased relative to the quasineutral flow to a desired potential, whereas in reality the potential would be set based on the operating conditions. Electrons being emitted from the leading edge of a hypersonic vehicle (cathode) and reattaching downstream (anode) is analogous to a double emissive probe. Emissive probes have been studied extensively in the plasma physics community. ${ }^{22}$ An important limit in this regime is space-charge limited emission. Space-charge limited emission occurs when there is a significant electron cloud in front of the emissive surface, which creates a virtual cathode that forces the electrons back to the surface. At the point at which the emission reaches this space-charge limit, there will be no virtual cathode and the electric field at the solid surface will be zero. The electrons are emitted cold for the case with zero electric field at the wall. As emission is increased, the virtual cathode will move off the surface and become larger to enforce the space-charge limit. A schematic of the sheath is shown in Fig. 1. If the emission is not space-charge limited, the potential within the sheath will be similar to the green curve. As emission is increased, it will reach the space-charge limit where the electric field is zero at the wall denoted by the blue curve. This type of emission will be referred to as cold emission. As emission is further increased, the virtual cathode will move off the wall and create a potential well as denoted by the red curve in Fig. 1. This will be referred to as warm emission because the emitted electrons will have a finite temperature. 


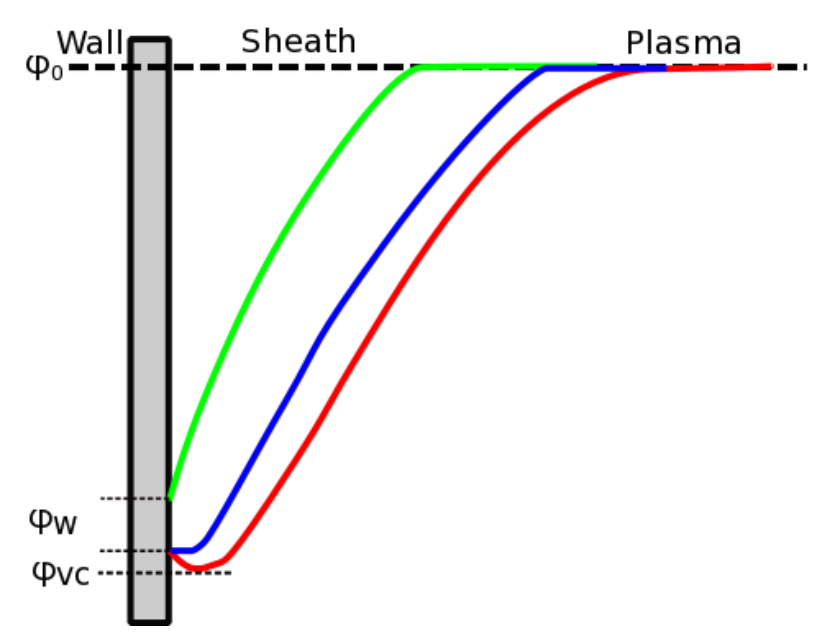

Figure 1: Sheath schematic: Richardson limited emission (green), space-charge limited cold emission (blue), and space-charge limited warm emission (red).

Takamura $^{23}$ et al. derived an expression for space-charge limited current through the sheath for the case with zero electric field at the surface and assuming that the ions arrive cold:

$$
\begin{gathered}
\frac{J_{e, \mathbf{e}}}{J_{i}}=\frac{G \sqrt{-\pi \Phi_{w}}}{1+G} \sqrt{\frac{2 m_{i}}{\pi m_{e}}} \\
\Phi_{w}=\frac{Q_{e}\left(\phi_{w}-\phi_{0}\right)}{T_{e}} \\
G=\frac{-\beta_{1}+\sqrt{\beta_{1}^{2}-4 \beta_{0} \beta_{2}}}{2 \beta_{2}} \\
\beta_{2}=-8 \Phi_{w}^{3}+4 \Phi_{w}^{2} \\
\beta_{1}=4 \Phi_{w}^{2}\left(1-2 \exp \Phi_{w}\right)+8 \Phi_{w}\left(\exp \Phi_{w}-1\right)-\left(\exp \Phi_{w}-1\right)^{2} \\
\beta_{0}=-4 \Phi_{w}^{2}-2 \Phi_{w}\left(\exp \Phi_{w}-1\right)\left(\exp \Phi_{w}-3\right)
\end{gathered}
$$

where $J_{i}$ is the ion saturation current defined by,

$$
J_{i}=Q_{e} n_{i} C_{s}
$$

It is to be noted that the potential at the sheath edge, $\phi_{0}$, is assumed to be zero. This theory has been compared to a 1-D PIC simulation and an experiment and agreed well. ${ }^{23}$ If the emitted electrons are not emitted cold and have a finite temperature, Equation 14 can be modified to account for this: ${ }^{23}$

$$
\begin{gathered}
\frac{J_{e, \mathbf{e}}}{J_{i}}=\frac{G^{*} \sqrt{-\pi \Phi_{v c}}}{1+A \cdot G^{*}} \sqrt{\frac{2 m_{i}}{\pi m_{e}}} \\
\Phi_{v c}=\frac{Q_{e}\left(\phi_{v c}-\phi_{0}\right)}{T_{e}} \\
G^{*}=f\left(\Phi_{v c}, \gamma\right) \\
A=\sqrt{\gamma \Phi_{v c}} \operatorname{erfc}\left(\gamma \Phi_{v c}\right) \exp \left(\gamma \Phi_{v c}\right) \\
\gamma=\frac{T_{e}}{T_{e, w}}
\end{gathered}
$$

where $\gamma$ is the ratio of electron temperature to emitted electron temperature. These relations will be compared to sheath results using a Direct Kinetic simulation. Equations 14 and 21 are implemented into LeMANS to account for space-charge effects and the results are discussed in Section III. 


\section{B. Grid-Based Direct Kinetic Simulation}

A grid-based direct kinetic method (DK) solver was developed at the University of Michigan. ${ }^{21}$ DK methods are an alternative approach to particle-based methods and are a more deterministic approach. A grid-based kinetic method employs discretized phase space in which the kinetic equations are solved directly. More details of the numerical approach can be found in Chapter II of Ref. 21.

\section{Numerical Results}

The benefits of ETC depend on the level of emission from the surface of the hypersonic vehicle, which places a high importance on modeling the process. An important phenomenon that can limit emission is a virtual cathode created by an electron cloud leading to space-charge limited emission. In the previous section, analytical models were presented that determine space-charge limited emission. A goal of this study is to assess the accuracy of the analytical space-charge limit relations and the assumptions built into them using a 1D direct kinetic solver. After assessing the analytical expressions, they are implemented into the CFD code, LeMANS, to perform an analysis of the effect the improved modeling of electron emission has on the leading edges of hypersonic vehicles, specifically the surface properties.

\section{A. Assessment of Analytical Sheath Models}

A test case is evaluated using the DK solver with the parameters given in Table 1. Helium (4 amu) was chosen as the ion and $\gamma$ as 10 because that was the fluid properties used in the 1D PIC verification of the analytical model. ${ }^{23} \frac{J_{e}, \mathbf{e}}{J_{i}}$ will be referred to as $\Gamma$, which describes the electron emission current relative to the ion current. $\Gamma_{\text {desired }}$ is the amount of emission that would result if the emission is not space-charge limited. The ratio of electron to ion temperature is typical to that of a hypersonic flow. This ratio is only used in the D-K simulations because the theory assumes cold ions. The sheath potential structure is shown in Fig. 2, where the potential is normalized by the electron temperature. Figure 2 shows that the emission is space-charge limited because of the virtual cathode shown near the wall (at $\mathrm{X}=0$ ). The potential then increases before reaching a plateau near the sheath edge (approximately $\mathrm{X}=15$ ). The $\Gamma_{\text {actual }}$ for this case is 21.7 , which is smaller than $\Gamma_{\text {desired }}$ since the emission is space-charge limited. Figure 3 shows the resulting velocity distribution function (VDF) for each species (ion, primary electron, and emitted electron) where the velocity is normalized by the ion acoustic speed.

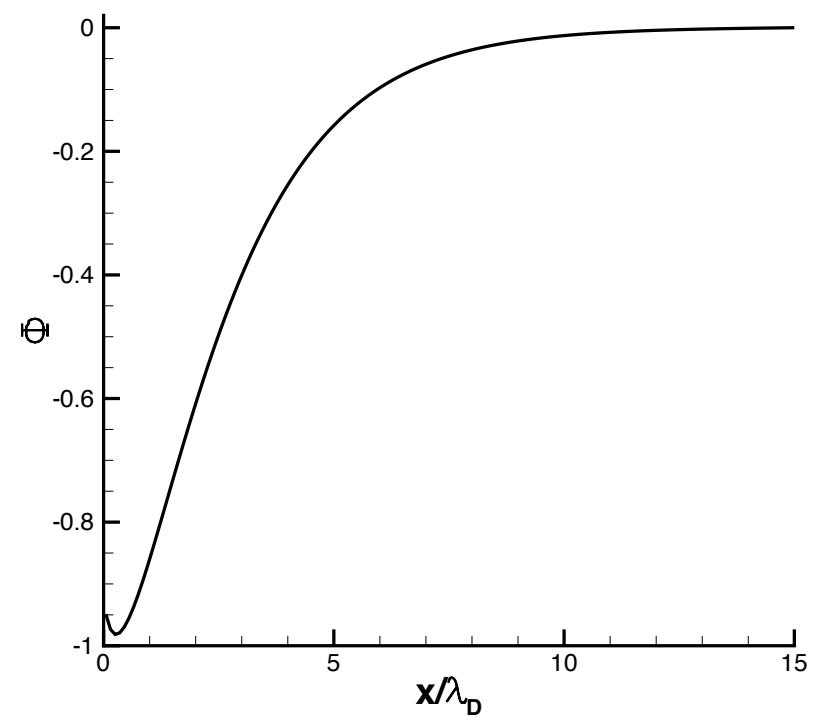

Figure 2: Sheath potential structure 
Table 1: Plasma sheath test case conditions

\begin{tabular}{ccccc}
\hline$\Phi_{w}$ & $\Gamma_{\text {desired }}$ & $m_{i}[\mathrm{amu}]$ & $\frac{T_{e}}{T_{i}}$ & $\gamma[\mathrm{EQ} 25]$ \\
\hline-0.95 & 50 & 4 & 10 & 10 \\
\hline
\end{tabular}

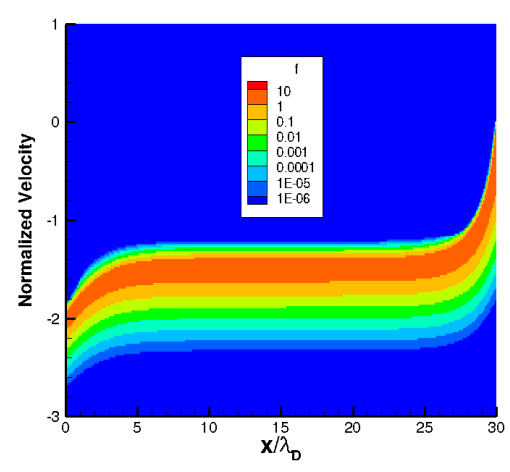

(a) Ions

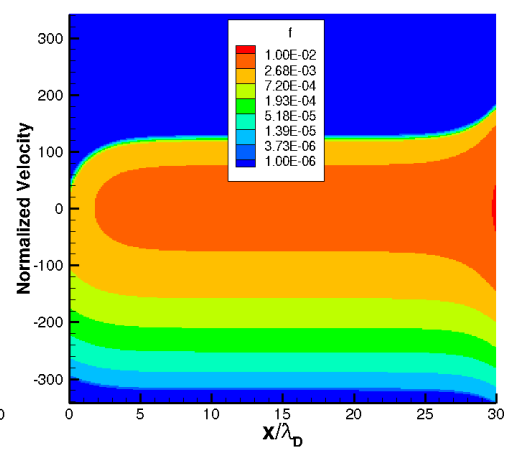

(b) Primary electrons

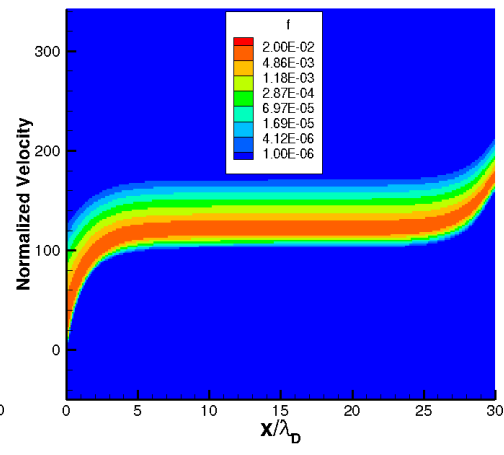

(c) Emitted electrons

Figure 3: Velocity distribution functions: $\mathrm{x}=0$ is the wall, $\mathrm{x}=30$ is the plasma, and the sheath edge is at the plateau (i.e. $\mathrm{x}=15$ )

\section{Theory: cold vs. warm emission}

To see the effect of temperature of the emitted electron has on space-charge limits, Eq. 14 is plotted against Eq. 21 for varying values of $\gamma$ in Fig. 4 for helium ( $4 \mathrm{amu}$ ). Figure 4 shows that cold emission results in a lower space-charge limit that with warm emission. This trend makes sense because electrons emitted with a finite temperature will have more energy to help overcome the virtual cathode. Electrons emitted with a higher finite temperature result in a higher space-charge limit as expected. This shows the importance of characterizing the temperature of the emitted electrons.

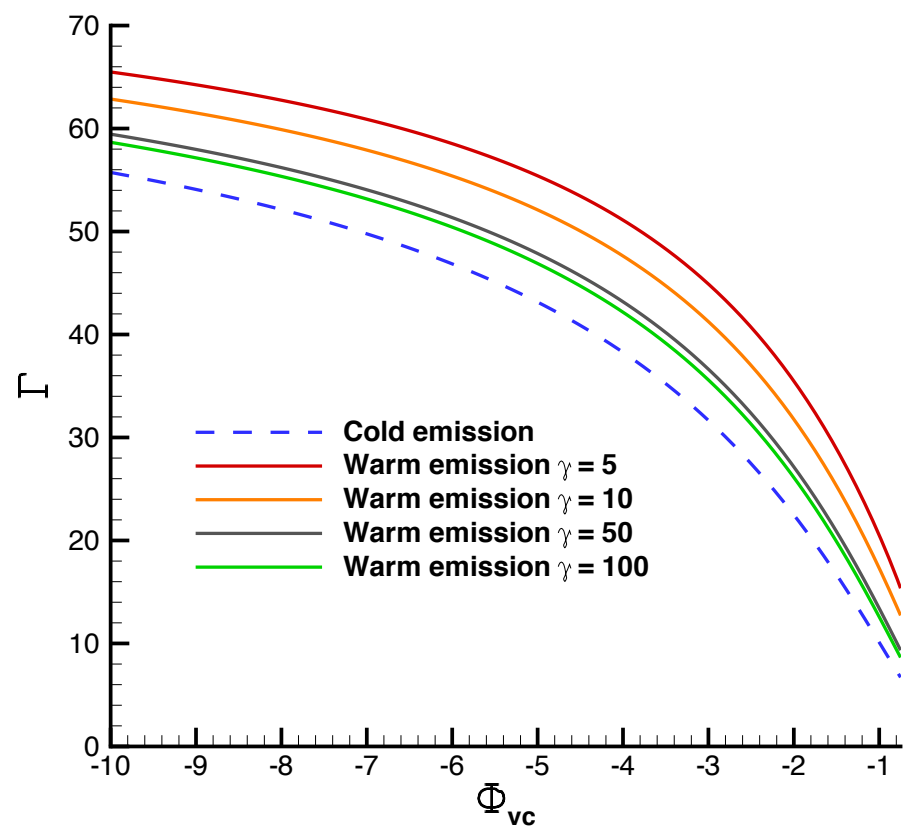

Figure 4: Cold vs. warm emitted electron temperature space-charge limited emission 


\section{Theory vs. direct kinetic solver}

The theory is evaluated using results obtained from the direct kinetic solver. Figure 5 shows a comparison between finite temperature theory (solid line) and results from the direct kinetic solver (symbols). For the range of low amount of wall bias (-1 to -3$)$, the theory under predicts the $\mathrm{D}-\mathrm{K}$ results. As the wall bias is increased in magnitude ( -3 to -10$)$, the theory over predicts the space-charge limited emission. For the D-K simulations that underpredicted the theory $(-3$ to -10$)$, oscillations were present due to instabilities such as two-stream instability. For the cases with oscillations, the resulting emission was time-averaged.

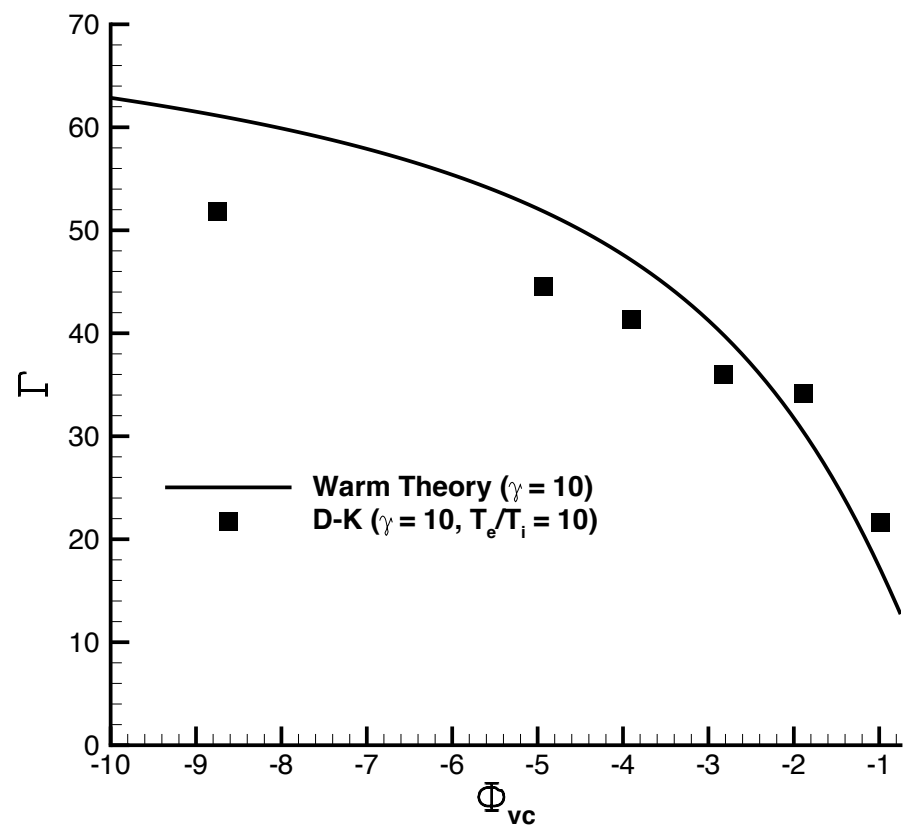

Figure 5: Comparison of space-charge limited theory to D-K simulations

\section{B. Electron Transpiration Cooling Test Case}

\section{Test Case Description}

The geometry of the test case considered in this study is representative of a $2 \mathrm{D}$ leading edge of a hypersonic vehicle and is shown in Fig. 6. The leading edge nose radius is $1.0 \mathrm{~cm}$, the wedge angle is 5.0 deg., and the length of the model is $25 \mathrm{~cm}$. The material radiative emissivity is assumed to equal 1.0. A mesh is generated for the geometry, and a grid convergence study revealed that the solution is grid-independent. Only one-half of the leading edge geometry is considered in the numerical simulations in order to reduce the computational cost using the axial vector as the line of symmetry. The computational grid contains approximately 20,000 cells, with 160 cells normal to the vehicle and 125 cells along the surface. The freestream conditions correspond to conditions at $60 \mathrm{~km}$ altitude and are listed in Table 2 . The freestream velocity is $6 \mathrm{~km} / \mathrm{s}$ and material work function is $2.0 \mathrm{eV}$.

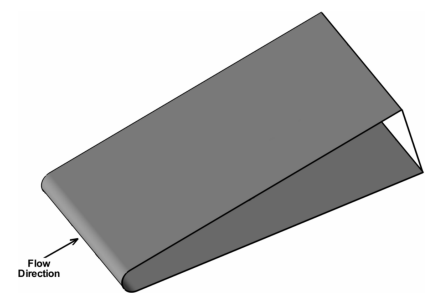

(a) 3-D View

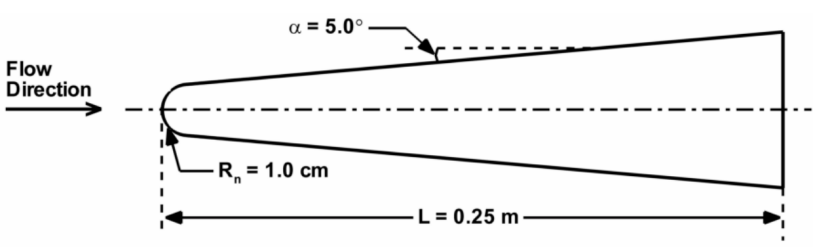

(b) Cross-Sectional View

Figure 6: Test case geometry 
Table 2: Freestream properties

\begin{tabular}{ccc}
\hline $\begin{array}{c}\text { Velocity, } \\
\mathrm{km} / \mathrm{s}\end{array}$ & $\begin{array}{c}\text { Temperature, } \\
\mathrm{K}\end{array}$ & $\begin{array}{c}\text { Density, } \\
\mathrm{kg} / \mathrm{m}^{3}\end{array}$ \\
\hline 6 & 238 & $2.30 \times 10^{-4}$ \\
\hline
\end{tabular}

\section{Flowfield features}

The flowfield features for the conditions typical of those investigated in this study are shown in Fig. 7, which show the temperature contours for the $6 \mathrm{~km} / \mathrm{s}$ case without ETC and with ETC $(\Phi=2.0 \mathrm{eV})$ and the temperature profiles along the stagnation line. For the case with ETC, the level of emission was determined by Richardson's current (Eq. 2) and space-charge effects were neglected. The flow for both cases is characterized by a strong bow shock that develops around the leading edge. The translational temperature rises to over 16,000 $\mathrm{K}$ across the shock before decreasing in the shock layer. The figure also shows that ETC has an overall small effect on the flowfield features. However, the shock standoff distance is slightly smaller and the surface temperature is lower for the case with electron emission.

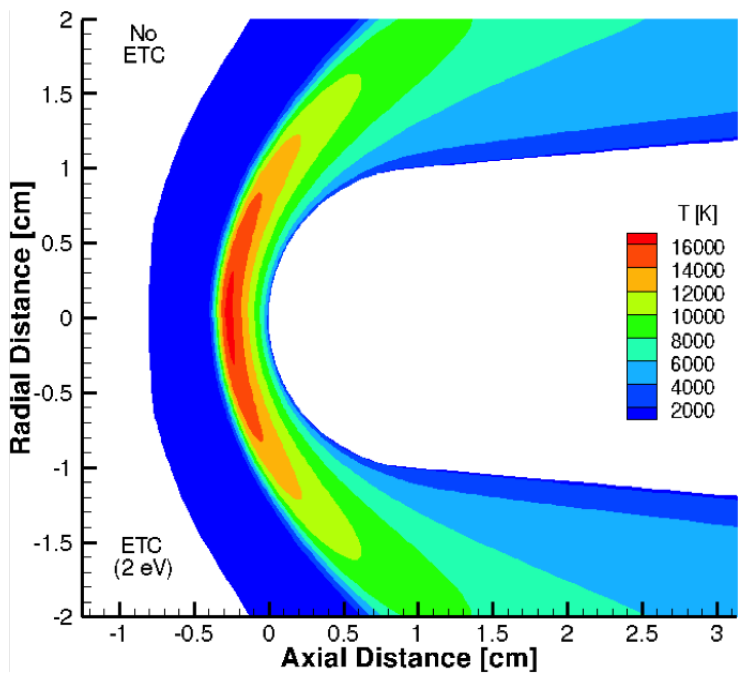

(a) Translational temperature contours: without ETC (top); with ETC(bottom)

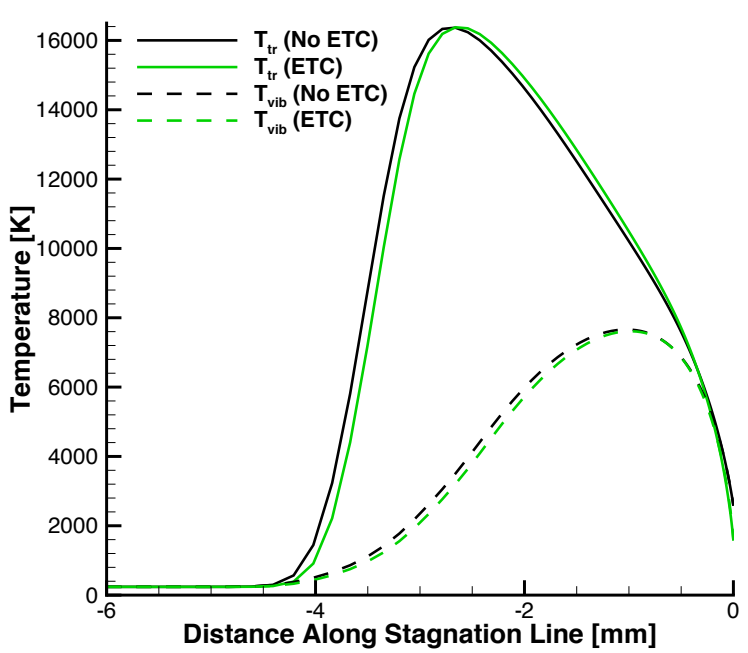

(b) Temperature profiles along stagnation line

Figure 7: Flowfield temperature contour and temperature profiles along the stagnation line

\section{Effect of ETC on Surface Properties}

The analytical expressions for the space-charge limited emission are implemented in LeMANS, and are evaluated for the conditions given in Table 2. Figure 8 presents the surface temperature profiles for different emission models. Note that the distance along the leading edge $(s)$ is normalized by the leading edge radius. The ideal ETC case assumes that there is no-space charge emission and the current is only limited by the surface temperature (Eq. 2) and results in about a $40 \%$ reduction in surface temperature. If space-charge limited emission is modeled assuming cold electron emission (Eq. 14), ETC results in approximately a $0.3 \%$ and $0.6 \%$ reduction in surface temperature for the -1 and -2 biased surface cases, respectively. It is to be noted that the surface temperature needs to be normalized by the electron temperature (wall temperature in this study) in order to compare to the potential plotted in Fig. 4. If space-charge limited emission is modeled assuming warm emission (Eq. 21), ETC results in approximately a $1 \%$ and $2 \%$ reduction in surface temperature for $\gamma=10$. If the temperature of the emitted is increased to $\gamma=5$, the benefits of decreases in surface temperature is magnified slightly. The reductions in surface temperature correspond to an increase 
in convective heat transfer as shown in Fig. 9. It is expected that a lower surface temperature corresponds to a higher convective heat transfer because the flowfield temperature is minimally changed by emission, and a larger temperature gradient will result in more convective heat transfer due to Fourier's Law. The emission current density for the cases with a bias of $-1 \mathrm{~V}$ is shown in Fig. 9. As expected the cases with the highest amount of current corresponds to the cases with the largest decrease in surface temperature. However, even with warm emission, the benefits of ETC are limited by space-charge limits. In previous work it was shown that the level of ionization in the flowfield can help overcome space-charge limits, ${ }^{8}$ so in future work, the test campaign will be expanded to higher velocities and less-sharp leading edge radii, as well as biasing the surface more.

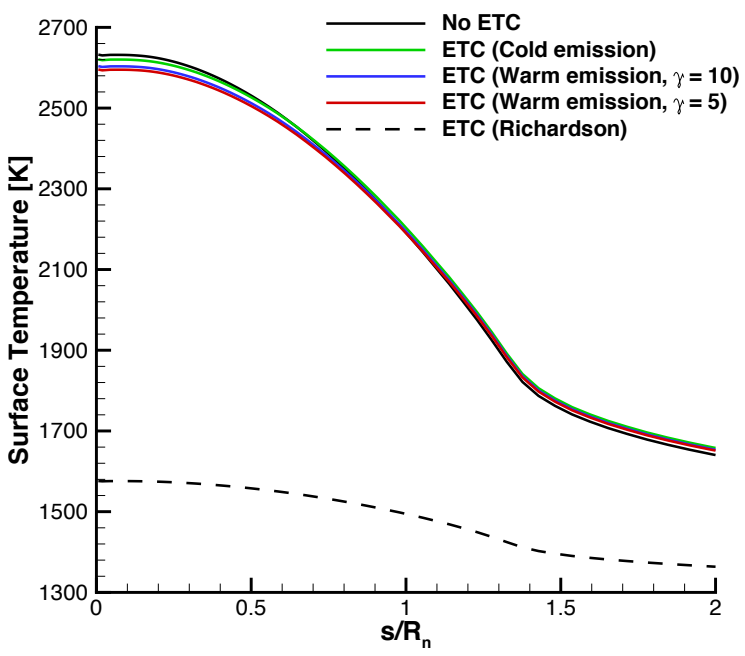

(a) Temperature $\left(\phi_{w / v c}=-1 \mathrm{~V}\right)$

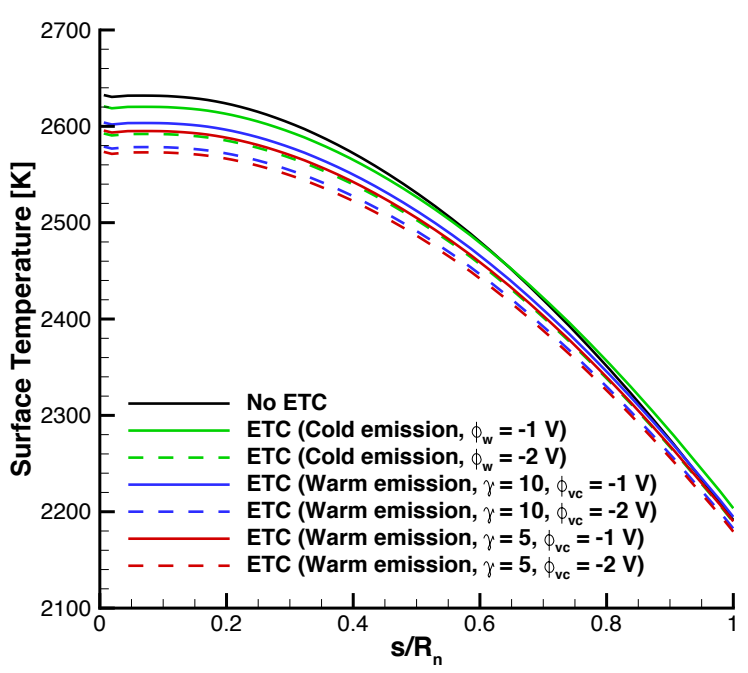

(b) Temperature (zoomed view)

Figure 8: Temperature surface profiles

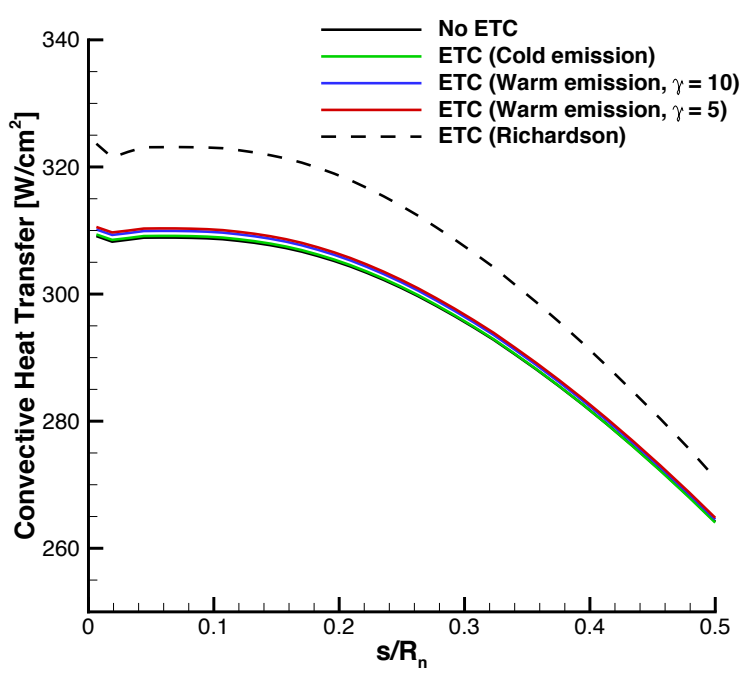

(a) Heat transfer

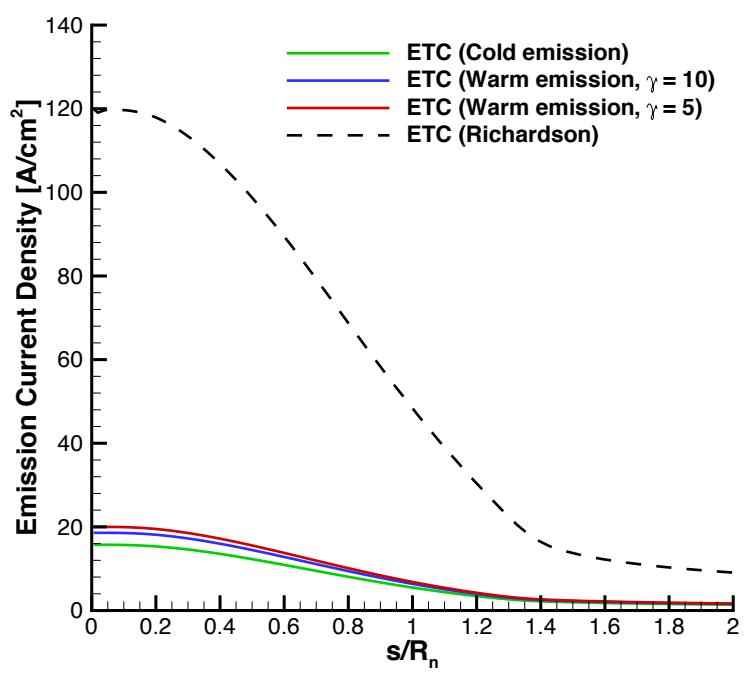

(b) Emission current density

Figure 9: Heat transfer and emission current density surface profiles $\left(\phi_{w / v c}=-1 \mathrm{~V}\right)$

\section{Conclusions and Future Work}

The goal of the present work was to investigate the effect plasma sheath physics has on electron transpiration cooling (ETC). Two different analytical models for space-charge limited emission were considered. 
The first model assumed that the electrons were emitted from the surface cold (cold emission). The second model assumed that the emitted electrons had a finite temperature (warm emission). The theory showed that electrons emitted with a finite electron temperature can reach higher levels of emission before a virtual cathode forms and pushes the electrons back to the surface. Both theories were assessed using 1D directkinetic simulation. The number of simulations were limited but those completed agreed well with the warm emission theory of Takamura et al. Finally, expressions from each theory were implemented into a CFD code, LeMANS, and applied to a test case typical of a leading edge radius in an hypersonic flight environment. The CFD results showed that the warm emission theory results in more emission than the cold emission theory, which generates a larger decrease in surface temperature. However, even with warm emission, the emission still reaches space-charge limits for the test case considered, which can limit the benefits of ETC.

Future work includes assessing the space-charge limit theory over a wider range of biased wall potentials, especially at more negatively biased potentials. The current work assumed a collisionless sheath, so future work will also include investigating the effect that collisions within the sheath would have on ETC. Future work will further investigate the effect of ETC on a full-scale hypersonic vehicle including collecting the emitted electrons downstream on the vehicle to complete the electrical circuit. Finally, experiments are needed to help validate the modeling approach.

\section{References}

\footnotetext{
${ }^{1}$ Fay, J. A. and Riddell, F. R., "Theory of Stagnation Point Heat Transfer in Dissociated Air," Journal of the Aeronautics Sciences, Vol. 25, 1958, pp. 73-85.

${ }^{2}$ Voland, R. T., Huebner, L. D., and McClinton, C. R., "X-43A Hypersonic Vehicle Technology Development," IAC-05D2.6.01, 2005.

${ }^{3}$ Glass, D. E., "Physical Challenges and Limitations Confronting the Use of UHTCs on Hypersonic Vehicles," AIAA Paper 2011-2304, 2011.

${ }^{4}$ Glass, D. E., "Ceramic Matrix Composite (CMC) Thermal Protection Systems (TPS) and Hot Structures for Hypersonic Vehicles," AIAA Paper 2008-2682, 2008.

${ }^{5}$ Uribarri, L. A. and Allen, E. H., "Electron Transpiration Cooling for Hot Aerospace Surfaces," AIAA Paper 2015-3674, July 2015.

${ }^{6}$ Alkandry, H., Hanquist, K. M., and Boyd, I. D., "Conceptual Analysis of Electron Transpiration Cooling for the Leading Edges of Hypersonic Vehicles," AIAA Paper 2014-2674, 2014.

${ }^{7}$ Hanquist, K. M., Alkandry, H., Boyd, I. D., "Evaluation of Computational Modeling of Electron Transpiration Cooling at High Enthalpies," AIAA Journal of Thermophysics and Heat Transfer, (accepted).

${ }^{8}$ Hanquist, K. M. and Boyd, I. D., "Limits for Thermionic Emission from Leading Edges of Hypersonic Vehicles," AIAA Paper 2016-0507, 2016.

${ }^{9}$ Martin, A., Scalabrin, L. C., and Boyd, I. D., "High Performance Modeling of Atmospheric Re-Entry Vehicles," Journal of Physics: Conference Series, Vol. 341, No. 1, 2012, Article 012002.

${ }^{10}$ Wilke, C. R., "A Viscosity Equation for Gas Mixtures," Journal of Chemical Physics, Vol. 18, No. 4, 1950, pp. 517-519.

${ }^{11}$ Vincenti, W. G. and Kruger, C. H., Introduction to Physical Gas Dynamics, Krieger Publishing Company, 2002.

${ }^{12}$ Blottner, F. G., Johnson, M., and Ellis, M., "Chemically Reacting Viscous Flow Program for Multi-Component Gas Mixtures," Tech. rep. SC-RR-70-754, Sandia Laboratories, Albuquerque, New Mexico, 1971.

${ }^{13}$ Park, C, Nonequilibrium Hypersonic Aerothermodynamics, John Wiley \& Sons, New York, 1990.

${ }^{14}$ MacCormack, R. W. and Candler, G. V., "The Solution of the Navier-Stokes Equations using Gauss-Seidel Line Relaxation," Computers and Fluids, Vol. 17, 1989, pp. 135-150.

${ }^{15}$ Richardson, O. W., The Emission of Electricity from Hot Bodies, Longmans, Green and Co., 1921.

${ }^{16}$ Scott, C. D., "Wall Catalytic Recombination and Boundary Conditions in Nonequilibrium Hypersonic Flows - with Applications," Advances in Hypersonics - Modeling Hypersonic Flows, Vol. 2, 1992, pp. 176-250.

${ }^{17}$ Razier, Y. P., Gas Discharge Physics, Springer-Verlag, 1991.

${ }^{18}$ Fridman, A. and Kennedy, L. A., Plasma Physics and Engineering, Taylor \& Francis, New York, 2004.

${ }^{19}$ Farbar, E., Boyd, I. D., and Martin, A., "Numerical Prediction of Hypersonic Flowfields Including Effects of Electron Translational Nonequilibrium," Journal of Thermophysics and Heat Transfer, Vol. 27, No.4, 2013, pp. 593-606.

${ }^{20}$ Sheridan, T. E. and Goree, J., "Collisional Plasma Sheath Model," Physics of Fluids B: Plasma Physics, Vol. 3, 1991, pp. 2796-2804.

${ }^{21}$ Hara, K., "Development of Grid-Based Direct Kinetic Method and Hybrid Kinetic Continuum Modeling of Hall Thruster Discharge Plasma," Ph. D. Dissertation, Department of Aerospace Engineering, University of Michigan, Ann Arbor, MI, 2015.

${ }^{22}$ Sheehan, J. P. and Hershkowitz, N., "Emissive Probes," Plasma Sources Science and Technology, Vol. 20, No. 6, 2011, pp. $1-22$.

${ }^{23}$ Takamura, S., Ohno, N., Ye, M. Y., and Kuwabara, T., "Space-Charge Limited Current from Plasma-Facing Material Surface," Contributions to Plasma Physics, Vol. 44, No. 1-3, 2004, pp. 126-137.
} 